\title{
Flavour and Colour of Quarks in Spin Topological Space
}

\author{
Shaoxu Ren \\ Institute of Physical Science and Engineering Tongji University, Shanghai, China \\ Email: shaoxu-ren@hotmail.com
}

How to cite this paper: Ren, S.X. (2021) Flavour and Colour of Quarks in Spin Topological Space. Journal of Modern Physics, 12, 380-389.

https://doi.org/10.4236/jmp.2021.123027

Received: January 14, 2021

Accepted: February 23, 2021

Published: February 26, 2021

Copyright ( 2021 by author(s) and Scientific Research Publishing Inc. This work is licensed under the Creative Commons Attribution International License (CC BY 4.0).

http://creativecommons.org/licenses/by/4.0/

\begin{abstract}
An assumption that all the six flavour quarks are attributed to be the components of a same, a common isospin multiplets space named STS is proposed. Base on Pauli Exclusion Principle, every quark is assigned to different flavour marks in STS. Every flavour quark possesses its own colour spectral line array specially appointed. The collection of colour spectral line arrays of the six flavour quarks constructs together the CSDF, Colour Spectrum Diagram of Flavour, further baryons and mesons could be constructed from CSDF. STS, Spin Topological Space is a math frame with infinite dimensional matrix representation for spin angular momentum. Flavours is an isospin angular momentum coupling phenomena of the three-colour-quarks.
\end{abstract}

\section{Keywords}

Pauli Exclusion Principle, STS, Spin Topological Space, STC, Spin Topological Coordinate, Colour Spectral Line Array, CSDF, Colour Spectrum Diagram of Flavour

\section{Introduction}

In isotopic spin space of Standard Model, SM, Gell-Mann M [1] and Zweig G [2], the isospin quantum number $I$ and the third component $I_{3}$, for flavour quarks $u, d$ are $1 / 2,+1 / 2$ and $1 / 2,-1 / 2$ respectively, for flavour quarks $s, c, b, t$ are $0,0 . u\left(I_{3}=+1 / 2\right)$ and $d\left(I_{3}=-1 / 2\right)$ quarks are assigned to an isodoublet with $I=1 / 2$, the dimension of matrix representation of the isodoublet is equal to $2 \times 1 / 2+1=2$. This matrix representation is an analogy with ordinary angular momentum $\vec{\sigma} / 2, \vec{\sigma}$ is Pauli matrix. And the remaining four flavour quarks $s\left(I_{3}=0\right), c\left(I_{3}=0\right), b\left(I_{3}=0\right), t\left(I_{3}=0\right)$ are assigned to four isosinglets with $I=0$, respectively, the dimension of matrix representation of each isosinglet is equal to $2 \times 0+1=1$. Further, there is an isodoublet and there are 
four isosinglets in isospin scheme for flavour quarks [3].

It is a curious question, what will happen? if the above six flavour quarks are all put into a common multiplet, that is, if these flavours are treated equally in one isotopic spin space. According to Pauli Exclusion Principle, PEP, each of those values of $I_{3}\left(q_{i}\right)$ of six flavour quarks should not be the same each other. Following the unified math symmetry picture, all eigenvalues of $I_{3}\left(q_{i}\right)$ of flavour $q_{i}$ quark are proposed to be half-integers, to be $+5 / 2,+3 / 2,+1 / 2,-1 / 2,-3 / 2$, $-5 / 2 . q_{i}=q_{t}, q_{c}, q_{u}, q_{d}, q_{s}, q_{b}, i=t, c, u, d, s, b$ respectively are shown in Table 1.

Table 1. Flavours quarks from SM to STS.

\begin{tabular}{|c|c|c|c|c|c|c|c|}
\hline Flavour & $I$ & $I_{3}\left(q_{i}\right)$ & matrix & PEP & $I$ & $I_{3}\left(q_{i}\right)$ & matrix \\
\hline Quark & SM & & & $\Rightarrow$ & STS & & infinite dimension \\
\hline$t$ & 0 & $I_{3}(t)=0$ & 1 dimension & & $1 / 2$ & $I_{3}(t)=+5 / 2$ & infinite dimension \\
\hline$c$ & 0 & $I_{3}(c)=0$ & 1 dimension & & $1 / 2$ & $I_{3}(c)=+3 / 2$ & infinite dimension \\
\hline$u$ & $1 / 2$ & $I_{3}(u)=+1 / 2$ & 2 dimension & & $1 / 2$ & $I_{3}(u)=+1 / 2$ & infinite dimension \\
\hline$d$ & $1 / 2$ & $I_{3}(d)=-1 / 2$ & 2 dimension & & $1 / 2$ & $I_{3}(d)=-1 / 2$ & infinite dimension \\
\hline$S$ & 0 & $I_{3}(s)=0$ & 1 dimension & & $1 / 2$ & $I_{3}(s)=-3 / 2$ & infinite dimension \\
\hline$b$ & 0 & $I_{3}(b)=0$ & 1 dimension & & $1 / 2$ & $I_{3}(b)=-5 / 2$ & infinite dimension \\
\hline
\end{tabular}

In Table 1, the right side is more graceful and elegant than the left side, but how can we obtain those third component eigenvalues $I_{3}\left(q_{i}\right)$ of isospin 1/2 particles, that labelled by mark $\$$, which are greater than $+1 / 2$ or less than $-1 / 2$ in Table 1? Next, we resort to Spin Topological Space ([4] [5] [6] [7]), abbreviation STS, that can help us to construct what we want to get the right side in Table 1.

\section{STS, Spin Topological Space}

Spin angular momentum $\vec{\pi}$ of a spin particle in STS math frame, is labelled by two subscripts $j, k$ (if in real region):

$$
\vec{\pi}_{j, k}=\left(\pi_{1 ; j, k}, \pi_{2 ; j, k}, \pi_{3 ; j, k}\right)
$$

$\vec{\pi}_{j, k}$ satisfy angular momentum commutation rule (2)

$$
\begin{gathered}
\vec{\pi}_{j, k} \times \vec{\pi}_{j, k}=i \vec{\pi}_{j, k} \\
\pi_{1 ; j, k}=\frac{1}{2}\left(\pi_{j}^{+}+\pi_{k}^{-}\right) \\
\pi_{2 ; j, k}=\frac{1}{2 i}\left(\pi_{j}^{+}-\pi_{k}^{-}\right) \\
\pi_{3 ; j, k}=\frac{1}{2}\left(\pi_{j}^{+} \pi_{k}^{-}-\pi_{k}^{-} \pi_{j}^{+}\right)
\end{gathered}
$$


$j, k \subset \mathrm{STS} . \pi_{1 ; j, k}$ and $\pi_{2 ; j, k}$ are two infinite dimensional Non-Hermitian Matrices. $\pi_{3 ; j, k}$ is an infinite dimensional Hermitian Matrix [4] [7]. Using the three components of $\vec{\pi}_{j, k}$, we get the expressions for the eigenvalue of Casimir Operator $\pi_{j, k}^{2}$ and the eigenvalue of the third component $\pi_{3 ; j, k}$ of $\vec{\pi}_{j, k}$ below

$$
\begin{gathered}
\pi_{j, k}^{2}=\pi_{1 ; j, k}^{2}+\pi_{2 ; j, k}^{2}+\pi_{3 ; j, k}^{2}=\frac{1}{4}\left\{(j-k)^{2}-1\right\} I_{0} \\
\pi_{3 ; j, k}=\pi_{0}(0)+\frac{1}{2}(j+k+1) I_{0} \\
\pi_{0}(0)=\operatorname{diag}\{\cdots, 5,4,3,2,1, \underline{0},-1,-2,-3,-4,-5, \cdots\} \\
I_{0}=\operatorname{diag}\{\cdots, 1,1,1,1,1, \underline{1}, 1,1,1,1,1, \cdots\}
\end{gathered}
$$

formulas (4), (5) show $\pi_{j, k}^{2}$ and $\pi_{3 ; j, k}$ are diagonal infinite dimensional matrices. Here $\pi_{0}(0)$ is the vacuum background spin angular momentum of $\pi_{3 ; j, k}$. If in case of no confusion, it is convenient to instead of (5) to use (9) to deal with $I_{3}$, then obtain following expressions

$$
\begin{gathered}
\pi_{j, k}^{2}=\frac{1}{4}\left(S_{j, k}^{2}-1\right) \\
\pi_{3 ; j, k}=\frac{1}{2}\left(A_{j, k}+1\right) \\
S_{j, k}=j-k, \quad A_{j, k}=j+k \\
(j, k)=\left(\frac{1}{2}\left(A_{j, k}+S_{j, k}\right), \frac{1}{2}\left(A_{j, k}-S_{j, k}\right)\right)
\end{gathered}
$$

Call $(j, k)$, STC, Spin Topological Coordinate of spin particle in STS.

Addition of $\vec{\pi}_{j, k}$ and $\vec{\pi}_{r, s}, \vec{\Pi}_{j, k ; r, s}$ is given below

$$
\begin{gathered}
\vec{\pi}_{j, k} \times \vec{\pi}_{j, k}=i \vec{\pi}_{j, k}, \quad \vec{\pi}_{r, s} \times \vec{\pi}_{r, s}=i \vec{\pi}_{r, s} \\
\vec{\Pi}_{j, k, r, s} \times \vec{\Pi}_{j, k, r, s}=i \vec{\Pi}_{j, k, k, s} \\
\vec{\Pi}_{j, k ; r, s}=\frac{1}{2}\left(\vec{\pi}_{j, k}+\vec{\pi}_{r, s}\right) \\
\Pi_{j, k ; r, s}^{2}=\frac{1}{16}\left(\left(S_{j, k}+S_{r, s}\right)^{2}-4\right)=\frac{1}{4}\left(\left(S_{j, k} / 2+S_{r, s} / 2\right)^{2}-1\right) \\
\Pi_{3 ; j, k, r, s}=\frac{1}{2}\left(\pi_{3 ; j, k}+\pi_{3 ; r, s}\right)
\end{gathered}
$$

$\Pi_{j, k, r, s}^{2}$ and $\Pi_{3 ; j, k ; r, s}$ are Casimir operator and the third component of spin particle $\vec{\Pi}_{j, k ; r, s}$ in STS.

\section{Flavour Quarks in STS}

Now we continue Table 1 quark model in STS, in flavour isotopic space, $\vec{\pi}$ is replaced by $\vec{I}\left(q_{i}\right)$, then obtain Casimir Operator $I^{2}\left(q_{i}\right)$ (18) and the third component eigenvalues $I_{3}\left(q_{i}\right)$ (19) of flavour $q_{i}$ quarks (fermion $I\left(q_{i}\right)=\frac{1}{2}$ (17)). Details are shown in Table 2. 
Table 2. Flavour quantum number of quarks in STS (isospin $I=\hbar / 2$ ).

\begin{tabular}{ccccc}
\hline$I_{3 ; j, k}\left(q_{i}\right)$ & $=\frac{1}{2}\left(A_{j, k}\left(q_{i}\right)+1\right)$ & $A_{j, k}\left(q_{i}\right)$ & $S_{j, k}\left(q_{i}\right)$ & $(j, k)_{q_{i}}$ \\
$I_{3 ;+3,+1}(t)=+5 / 2^{*}$ & $\frac{1}{2}(+4+1)$ & $A_{+3,+1}(t)=+4$ & $S_{+3,+1}(t)=+2$ & $(+3,+1)_{t}$ \\
$I_{3 ;+2,0}(c)=+3 / 2^{*}$ & $\frac{1}{2}(+2+1)$ & $A_{+2,0}(c)=+2$ & $S_{+2,0}(c)=+2$ & $(+2,0)_{c}$ \\
$I_{3 ;+1,-1}(u)=+1 / 2$ & $\frac{1}{2}(0+1)$ & $A_{+1,-1}(u)=0$ & $S_{+1,-1}(u)=+2$ & $(+1,-1)_{u}$ \\
$I_{3 ; 0,-2}(d)=-1 / 2$ & $\frac{1}{2}(-2+1)$ & $A_{0,-2}(d)=-2$ & $S_{0,-2}(d)=+2$ & $(0,-2)_{d}$ \\
$I_{3 ;-1,-3}(s)=-3 / 2^{*}$ & $\frac{1}{2}(-4+1)$ & $A_{-1,-3}(s)=-4$ & $S_{-1,-3}(s)=+2$ & $(-1,-3)_{s}$ \\
$I_{3 ;-2,-4}(b)=-5 / 2^{*}$ & $\frac{1}{2}(-6+1)$ & $A_{-2,-4}(b)=-6$ & $S_{-2,-4}(b)=+2$ & $(-2,-4)_{b}$
\end{tabular}

Note: $A_{j, k}\left(q_{i}\right)$ is named as flavour quantum number of quarks, which are even numbers.

$$
\begin{gathered}
I\left(q_{i}\right)=\frac{1}{2} \\
I_{j, k}^{2}\left(q_{i}\right)=\operatorname{diag}\left\{\cdots, \frac{+3}{4}, \frac{+3}{4}, \frac{+3}{4}, \frac{+3}{4}, \frac{+3}{4}, \frac{+3}{4}, \frac{+3}{4}, \cdots\right\} \\
I_{3 ; j, k}\left(q_{i}\right)=\operatorname{diag}\left\{\cdots, \frac{+7}{2} \bullet, \frac{+5}{2} \bullet, \frac{+3}{2} \bullet, \frac{+1}{2}, \frac{-1}{2}, \frac{-3}{2} \bullet, \frac{-5}{2} \bullet, \cdots\right\}, i=t, c, u, d, s, b
\end{gathered}
$$

\section{Colour Quarks in STS}

Now Suppose that except flavour quantum number marked $A_{j, k}\left(q_{i}\right)$ in STS (Table 2), quark could even possess colour quantum number array that called as Colour Spectral Line Array labelled by $q_{\mathrm{RGB}}(20)$, which is an array comprised of three colour quantum numbers, marked $q_{\mathrm{R}}, q_{\mathrm{G}}$ and $q_{\mathrm{B}}$, they are third-fractions.

$$
q_{\mathrm{RGB}} \equiv\left(q_{\mathrm{R}}, q_{\mathrm{G}}, q_{\mathrm{B}}\right) \equiv\left(A\left(q_{\mathrm{R}}\right), A\left(q_{\mathrm{G}}\right), A\left(q_{\mathrm{B}}\right)\right)
$$

Different flavour quark possesses its own colour spectral line array, for example, array $u_{\mathrm{RGB}} \equiv\left(u_{\mathrm{R}}, u_{\mathrm{G}}, u_{\mathrm{B}}\right)=\left(\frac{+2}{3}, \frac{+5}{3}, \frac{+11}{3}\right)$ is the colour spectral line array of flavour $u$ quark, and array $d_{\mathrm{RGB}} \equiv\left(d_{\mathrm{R}}, d_{\mathrm{G}}, d_{\mathrm{B}}\right)=\left(\frac{-16}{3}, \frac{-13}{3}, \frac{-7}{3}\right)$ is colour spectral line array of flavour $d$ quark. Flavour $A_{j, k}\left(q_{i}\right)$ and colour $q_{\mathrm{RGB}}$ are the identities of quark particles.

Definition CSDF, Colour Spectrum Diagram of Flavour is composed of six (or more) colour spectral line arrays of flavour quarks, somewhat similar to the Gene diagram of chromosomes. Explicit scheme of CSDF is given below. 
Quark

$I_{3}\left(q_{\mathrm{RGB}}\right)$

$$
t, \frac{+5}{2}
$$

$c, \frac{+3}{2}$

$u, \frac{+1}{2}$

$d, \frac{-1}{2}$

$s, \frac{-3}{2}$

$b, \frac{-5}{2}$

$I_{3}\left(t_{\mathrm{R}}, t_{\mathrm{G}}, t_{\mathrm{B}}\right)$

$I_{3}\left(c_{\mathrm{R}}, c_{\mathrm{G}}, c_{\mathrm{B}}\right)$

$I_{3}\left(u_{\mathrm{R}}, u_{\mathrm{G}}, u_{\mathrm{B}}\right)$

$I_{3}\left(d_{\mathrm{R}}, d_{\mathrm{G}}, d_{\mathrm{B}}\right)$

$I_{3}\left(s_{\mathrm{R}}, s_{\mathrm{G}}, s_{\mathrm{B}}\right)$

$I_{3}\left(b_{\mathrm{R}}, b_{\mathrm{G}}, b_{\mathrm{B}}\right)$

$\left(\frac{+41}{6}, \frac{+44}{6}, \frac{+50}{6}\right) \quad\left(\frac{+23}{6}, \frac{+26}{6}, \frac{+32}{6}\right)$

$\left(\frac{+5}{6}, \frac{+8}{6}, \frac{+14}{6}\right)$

$\left(\frac{-13}{6}, \frac{-10}{6}, \frac{-4}{6}\right)$

$\left(\frac{-31}{6}, \frac{-28}{6}, \frac{-22}{6}\right) \quad\left(\frac{-49}{6}, \frac{-46}{6}, \frac{-40}{6}\right)$

$\bar{t}, \frac{-5}{2}$

$\bar{c}, \frac{-3}{2}$

$\bar{u}, \frac{-1}{2}$

$\bar{d}, \frac{+1}{2}$

$\bar{s}, \frac{+3}{2}$

$\bar{b}, \frac{+5}{2}$

Anti-Quark

$I_{3}\left(\bar{t}_{\overline{\mathrm{R}}}, \overline{t_{\overline{\mathrm{G}}}}, \overline{\overline{\mathrm{B}}_{\overline{\mathrm{B}}}}\right)$

$I_{3}\left(\bar{c}_{\overline{\mathrm{R}}}, \bar{c}_{\overline{\mathrm{G}}}, \bar{c}_{\overline{\mathrm{B}}}\right)$

$I_{3}\left(\bar{u}_{\overline{\mathrm{R}}}, \bar{u}_{\overline{\mathrm{G}}}, \bar{u}_{\overline{\mathrm{B}}}\right)$

$I_{3}\left(\bar{d}_{\overline{\mathrm{R}}}, \bar{d}_{\overline{\mathrm{G}}}, \bar{d}_{\overline{\mathrm{B}}}\right)$

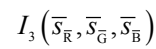

$I_{3}\left(\bar{b}_{\overline{\mathrm{R}}}, \bar{b}_{\overline{\mathrm{G}}}, \bar{b}_{\overline{\mathrm{B}}}\right)$

$\left(\frac{-41}{6}, \frac{-44}{6}, \frac{-50}{6}\right) \quad\left(\frac{-23}{6}, \frac{-26}{6}, \frac{-32}{6}\right)$

$\left(\frac{-5}{6}, \frac{-8}{6}, \frac{-14}{6}\right)$

$\left(\frac{+13}{6}, \frac{+10}{6}, \frac{+4}{6}\right)$

$\left(\frac{+31}{6}, \frac{+28}{6}, \frac{+22}{6}\right) \quad\left(\frac{+49}{6}, \frac{+46}{6}, \frac{+40}{6}\right)$
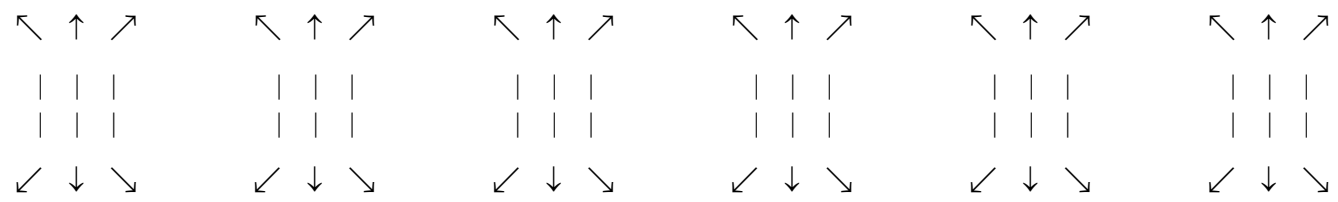

$t,+4$

$c,+2$

$u, 0$

$d,-2$

$s,-4$

$b,-6$

Quark

$\left(t_{\mathrm{R}}, t_{\mathrm{G}}, t_{\mathrm{B}}\right)$

$\left(c_{\mathrm{R}}, c_{\mathrm{G}}, c_{\mathrm{B}}\right)$

$\left(u_{\mathrm{R}}, u_{\mathrm{G}}, u_{\mathrm{B}}\right)$

$\left(d_{\mathrm{R}}, d_{\mathrm{G}}, d_{\mathrm{B}}\right)$

$\left(s_{\mathrm{R}}, s_{\mathrm{G}}, s_{\mathrm{B}}\right)$

$\left(b_{\mathrm{R}}, b_{\mathrm{G}}, b_{\mathrm{B}}\right)$

$q_{\mathrm{RGB}}$

$\left(\frac{+38}{3}, \frac{+41}{3}, \frac{+47}{3}\right)$

$\left(\frac{+20}{3}, \frac{+23}{3}, \frac{+29}{3}\right) \quad\left(\frac{+2}{3}, \frac{+5}{3}, \frac{+11}{3}\right)$

$\left(\frac{-16}{3}, \frac{-13}{3}, \frac{-7}{3}\right)$

$\left(\frac{-34}{3}, \frac{-31}{3}, \frac{-25}{3}\right) \quad\left(\frac{-52}{3}, \frac{-49}{3}, \frac{-43}{3}\right)$

$\bar{t},-6$

$\bar{c},-4$

$\bar{u},-2$

$\bar{d}, 0$

$\bar{s},+2$

$\bar{b},+4$

Anti-Quark

$\left(\bar{t}_{\mathrm{R}}, \bar{t}_{\mathrm{G}}, \overline{\mathrm{B}}_{\mathrm{B}}\right)$

$\left(\bar{c}_{\overline{\mathrm{R}}}, \bar{c}_{\overline{\mathrm{G}}}, \bar{c}_{\overline{\mathrm{B}}}\right)$

$\left(\bar{u}_{\overline{\mathrm{R}}}, \bar{u}_{\overline{\mathrm{G}}}, \bar{u}_{\overline{\mathrm{B}}}\right)$

$\left(\bar{d}_{\overline{\mathrm{R}}}, \bar{d}_{\overline{\mathrm{G}}}, \bar{d}_{\overline{\mathrm{B}}}\right)$

$\left(\overline{s_{\overline{\mathrm{R}}}}, \overline{s_{\overline{\mathrm{G}}}}, \overline{s_{\overline{\mathrm{B}}}}\right)$

$\left(\bar{b}_{\overline{\mathrm{R}}}, \bar{b}_{\overline{\mathrm{G}}}, \bar{b}_{\overline{\mathrm{B}}}\right)$

$\bar{q}_{\overline{\mathrm{R}} \overline{\mathrm{B}} \overline{\mathrm{B}}}$

$\left(\frac{-44}{3}, \frac{-47}{6}, \frac{-53}{6}\right) \quad\left(\frac{-26}{3}, \frac{-29}{3}, \frac{-35}{3}\right)$

$\left(\frac{-8}{3}, \frac{-11}{3}, \frac{-17}{3}\right)$

$\left(\frac{+10}{3}, \frac{+7}{3}, \frac{+1}{3}\right)$

$\left(\frac{+28}{3}, \frac{+25}{3}, \frac{+19}{3}\right) \quad\left(\frac{+46}{3}, \frac{+43}{3}, \frac{+37}{3}\right)$

\section{Hypothesis}

Flavours are coupling phenomena of isospin angular momenta of threecolour-quarks.

To track the idea, we make use of CSDF, and of angular momentum formulae (21) and (22) of three spin particles below. Further obtain STC array of flavour quarks in Table 3. In other words, obtain the relationships STC array,

$(j, k)_{q_{\mathrm{i}}}=\left((j, k)_{q_{\mathrm{R}}},(j, k)_{q_{\mathrm{G}}},(j, k)_{q_{\mathrm{B}}}\right)$ between flavour quantum number, $A_{j, k}\left(q_{i}\right)$ and Colour Spectral Line Array $q_{\mathrm{RGB}}=\left(q_{\mathrm{R}}, q_{\mathrm{G}}, q_{\mathrm{B}}\right)$.

$$
\begin{gathered}
I^{2}(3 q)=\frac{1}{36}\left(\left(S\left(q_{\mathrm{R}}\right)+S\left(q_{\mathrm{R}}\right)+S\left(q_{\mathrm{B}}\right)\right)^{2}-9\right)=\frac{3}{4} \\
I_{3}(3 q)=\frac{1}{3}\left(I_{3}\left(q_{\mathrm{R}}\right)+I_{3}\left(q_{\mathrm{G}}\right)+I_{3}\left(q_{\mathrm{B}}\right)\right)=\frac{1}{2}\left(\frac{A(3 q)}{3}+1\right) \\
I_{3}(q)=\overline{I_{3}(3 q)}=\frac{1}{3} I_{3}(3 q)
\end{gathered}
$$


Table 3. STC array of colour quantum numbers of flavour quarks.

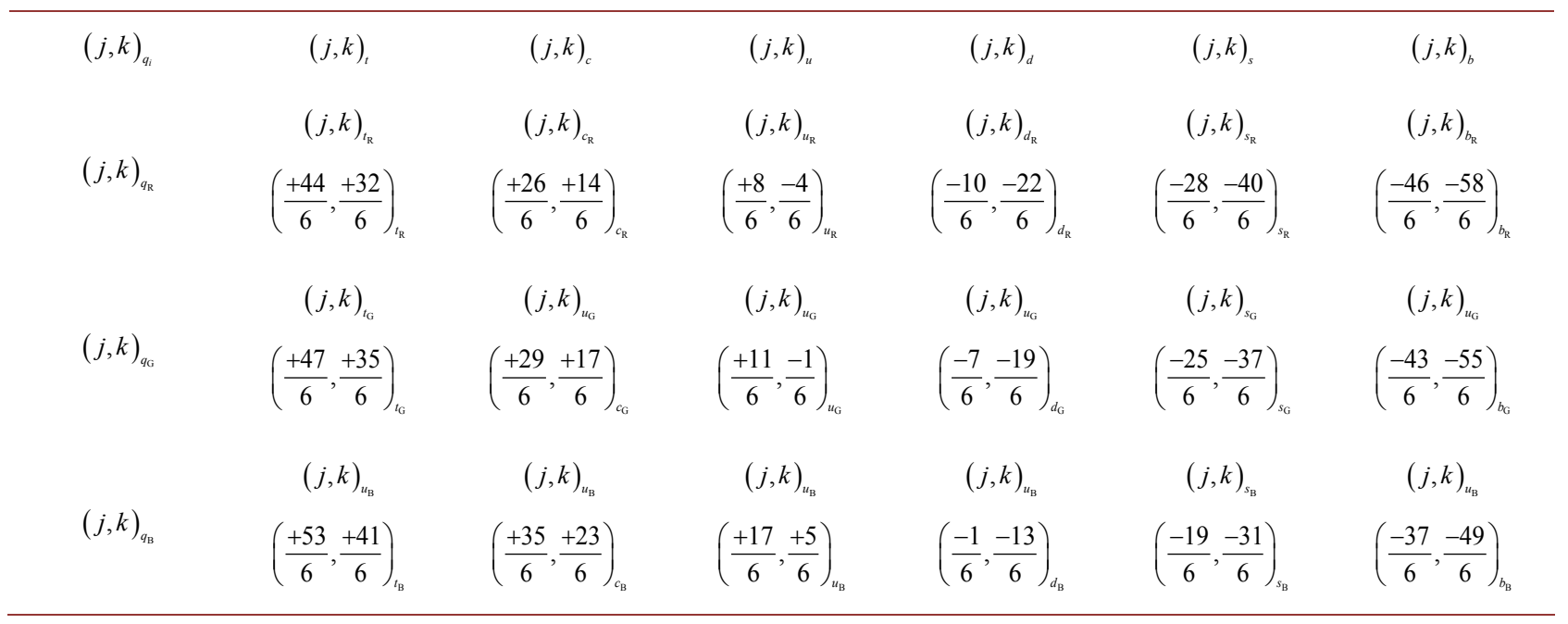

\section{Baryons and Mesons in STS}

Due to baryons all are "white colour" particles, which are made of colourful quarks. To help with CSDF, picking up three colour quantum numbers: $q_{\mathrm{R}}^{1} \subseteq q_{\mathrm{RGB}}^{1}$, $q_{\mathrm{G}}^{2} \subseteq q_{\mathrm{RGB}}^{2}$ and $q_{\mathrm{B}}^{3} \subseteq q_{\mathrm{RGB}}^{3}$, respectively from any three quarks $q^{1}, q^{2}$ and $q^{3}\left(q^{1}\right.$, $q^{2}$ and $q^{3}$ can be any flavour), then various visible baryons could be produced. In this way, for example, baryon decuplet is constituted as shown in Table 4.

According to SM, the "colourless phenomena" of all mesons could be satisfied by blending with a quark with colour and antiquark with anti-colour, that is to say, $q_{\mathrm{R}} \bar{q}_{\overline{\mathrm{R}}}$, or $q_{\mathrm{G}} \bar{q}_{\overline{\mathrm{G}}}$, or $q_{\mathrm{B}} \bar{q}_{\overline{\mathrm{B}}}$. Contrary to SM, in STS a meson is similar to a baryon, a meson also is a three-body system that comprises a quark (colour), an antiquark (anti-colour) and a gluon (white). This gluon plays the role of mediator to fasten quark and antiquark together in a meson.

Colour spectral line array of meson is symbolized with $q_{i} \bar{q}_{\bar{j}} g_{k}=\left(q_{i}, \bar{q}_{\bar{j}}, g_{k}\right)$. The mentioned above is the case of $i=j, k=0$. The discussion about $i \neq j$, $k \neq 0 \quad$ will be given later.

In what follows base on CSDF, we list the weight diagram Table 5 of meson octet. Here $g_{0}$ is the gluon basic state with $I_{3}^{2}\left(g_{0}\right)=0 \hbar, A\left(g_{0}\right)=-1$.

\section{7. $\vec{A}(q) \cdot \vec{A}(q)$ Interaction in STS}

This paragraph suggests some ideas, similar to spin-spin $\overrightarrow{S_{i}} \cdot \overrightarrow{S_{j}}$ interaction in spin space [3], to disscuss $\vec{A}(q) \cdot \vec{A}(q)$ Interaction in STS. Table 4 shows that baryons, like quarks (CSDF), are marked by colour spectral line arrays too, but a slight different from quarks. Actually, there are two kinds of colour spectral line arrays: right-hand colour quantum numbers $(r-h)(23.1)$ and left-hand colour quantum numbers (1-h) (23.2) for a given baryon, which made of quark $q^{1}, q^{2}$ and $q^{3}$. Each baryon exists in one of three possible states, labelled with cases: I, II and III in case (23.1) and case (23.2) respectively. The results in Table 4 are the case of I of $(r-h)$ only shown below. 
Table 4. Weight diagram for baryon decuplet with $S=+4$ in STS.

$$
\begin{aligned}
& I_{3}\left(\Delta^{-}\right)=\frac{-3}{2},-4 \\
& I_{3}\left(\Delta^{0}\right)=\frac{-1}{2},-2 \\
& \left(d_{\mathrm{R}}, d_{\mathrm{G}}, d_{\mathrm{B}}\right) \\
& \left(u_{\mathrm{R}}, d_{\mathrm{G}}, d_{\mathrm{B}}\right) \\
& \left(\frac{-16}{3}, \frac{-13}{3}, \frac{-7}{3}\right) \\
& \left(\frac{+2}{3}, \frac{-13}{3}, \frac{-7}{3}\right) \\
& \left(u_{\mathrm{R}}, d_{\mathrm{G}}, u_{\mathrm{B}}\right) \\
& \left(\frac{+2}{3}, \frac{-13}{3}, \frac{+11}{3}\right) \\
& \begin{array}{c}
I_{3}\left(\Delta^{++}\right)=\frac{+3}{2},+2 \\
\left(u_{\mathrm{R}}, u_{\mathrm{G}}, u_{\mathrm{B}}\right) \\
\left(\frac{+2}{3}, \frac{+5}{3}, \frac{+11}{3}\right)
\end{array} \\
& \begin{array}{c}
I_{3}\left(\Sigma^{*-}\right)=\frac{-5}{2},-6 \\
\left(d_{\mathrm{R}}, s_{\mathrm{G}}, d_{\mathrm{B}}\right) \\
\left(\frac{-16}{3}, \frac{-31}{3}, \frac{-7}{3}\right)
\end{array} \\
& I_{3}\left(\Sigma^{* 0}\right)=\frac{-3}{2},-4 \\
& \left(u_{\mathrm{R}}, s_{\mathrm{G}}, u_{\mathrm{B}}\right) \\
& \left(\frac{+2}{3}, \frac{-31}{3}, \frac{-7}{3}\right) \\
& I_{3}\left(\Sigma^{*+}\right)=\frac{-1}{2},-2 \\
& \left(u_{\mathrm{R}}, s_{\mathrm{G}}, u_{\mathrm{B}}\right) \\
& \left(\frac{+2}{3}, \frac{-31}{3}, \frac{+11}{3}\right) \\
& I_{3}\left(\Xi^{*-}\right)=\frac{-7}{2},-8 \\
& \left(d_{\mathrm{R}}, s_{\mathrm{G}}, s_{\mathrm{B}}\right) \\
& \left(\frac{-16}{3}, \frac{-31}{3}, \frac{-25}{3}\right) \\
& \begin{array}{c}
I_{3}\left(\Xi^{* 0}\right)=\frac{-5}{2},-6 \\
\left(u_{\mathrm{R}}, s_{\mathrm{G}}, s_{\mathrm{B}}\right) \\
\left(\frac{+2}{3}, \frac{-31}{3}, \frac{-25}{3}\right)
\end{array} \\
& I_{3}\left(\Omega^{-}\right)=\frac{-9}{2},-10 \\
& \left(s_{\mathrm{R}}, s_{\mathrm{G}}, s_{\mathrm{B}}\right) \\
& \left(\frac{-34}{3}, \frac{-31}{3}, \frac{-25}{3}\right)
\end{aligned}
$$

Note: in Table 4, the value $I_{3}$ of every baryon all is half integer, contrary to those what the $I_{3}$ might take both half integer and integer (include zero) in case of $J^{P}=3 / 2^{+}$in SM.

Table 5. Weight diagram for meson octet with $S=+1$ in STS.

$$
\begin{gathered}
I_{3}\left(k^{0}\right)=+1, A\left(k^{0}\right)=+1 \\
\left(d_{\mathrm{R}}, \bar{s}_{\overline{\mathrm{R}}}, g_{0}\right) \\
\left(\frac{-16}{3}, \frac{+28}{3}, \frac{-3}{3}\right)
\end{gathered}
$$

$$
\begin{gathered}
I_{3}\left(k^{+}\right)=+2, A\left(k^{+}\right)=+3 \\
\left(u_{\mathrm{R}}, \bar{s}_{\overline{\mathrm{R}}}, g_{0}\right) \\
\left(\frac{+2}{3}, \frac{+28}{3}, \frac{-3}{3}\right)
\end{gathered}
$$

$$
\begin{array}{ccc}
\begin{array}{l}
I_{3}\left(\pi^{-}\right)=-1, A\left(\pi^{-}\right)=-3 \\
\left(d_{\mathrm{R}}, \bar{u}_{\overline{\mathrm{R}}}, g_{0}\right)
\end{array} & I_{3}\left(\pi^{0}, \eta\right)=0, A\left(\pi^{0}, \eta\right)=-1 & I_{3}\left(\pi^{+}\right)=+1, A\left(\pi^{+}\right)=+1 \\
\left(\frac{-16}{3}, \frac{-8}{3}, \frac{-3}{3}\right) & \left(u_{\mathrm{R}}, \bar{u}_{\overline{\mathrm{R}}}, g_{0}\right) & \left(u_{\mathrm{R}}, \bar{d}_{\overline{\mathrm{R}}}, g_{0}\right) \\
& \left(\frac{+2}{3}, \frac{-8}{3}, \frac{-3}{3}\right) & \left(\frac{+2}{3}, \frac{+10}{3}, \frac{-3}{3}\right) \\
& \left(\frac{-16}{3}, \frac{+10}{3}, \frac{-3}{3}\right) & \\
I_{3}\left(k^{-}\right)=-2, A\left(k^{-}\right)=-5 & \left.g_{0}\right) & I_{3}\left(\bar{k}^{0}\right)=-1, A\left(\bar{k}^{0}\right)=-3 \\
\left(s_{\mathrm{R}}, \bar{u}_{\overline{\mathrm{R}}}, g_{0}\right) & \left(s_{\mathrm{R}}, \bar{d}_{\overline{\mathrm{R}}}, g_{0}\right) \\
& \left(\frac{-34}{3}, \frac{-8}{3}, \frac{-3}{3}\right) & \left(\frac{-34}{3}, \frac{+10}{3}, \frac{-3}{3}\right)
\end{array}
$$

Note: in Table 5, the value $I_{3}$ of every meson all is integer, contrary to those what the $I_{3}$ might take both half integer and integer in case of $J^{P}=0^{-}$in SM. 
There is an amusing equality (23) below among $q^{1}, q^{2}$ and $q^{3}$ that is obtained from $q_{\mathrm{RGB}} \equiv\left(q_{\mathrm{R}}, q_{\mathrm{G}}, q_{\mathrm{B}}\right)$

$$
\begin{aligned}
\mathrm{IV}(r-h) \cdot q^{1}+q^{2}+q^{3} & \equiv \mathrm{I} \cdot q_{\mathrm{R}}^{1}+q_{\mathrm{G}}^{2}+q_{\mathrm{B}}^{3}=\mathrm{II} \cdot q_{\mathrm{R}}^{2}+q_{\mathrm{G}}^{3}+q_{\mathrm{B}}^{1}=\mathrm{III} \cdot q_{\mathrm{R}}^{3}+q_{\mathrm{G}}^{1}+q_{\mathrm{B}}^{2}, \\
A & =\frac{1}{3}\left(q^{1}+q^{2}+q^{3}\right) \\
\mathrm{IV}(l-h) \cdot q^{2}+q^{1}+q^{3} & \equiv \mathrm{I} \cdot q_{\mathrm{R}}^{2}+q_{\mathrm{G}}^{1}+q_{\mathrm{B}}^{3}=\mathrm{II} \cdot q_{\mathrm{R}}^{3}+q_{\mathrm{G}}^{2}+q_{\mathrm{B}}^{1}=\mathrm{III} \cdot q_{\mathrm{R}}^{1}+q_{\mathrm{G}}^{3}+q_{\mathrm{B}}^{2}, \\
A & =\frac{1}{3}\left(q^{2}+q^{1}+q^{3}\right)
\end{aligned}
$$

example of $p^{+}$

$$
\begin{aligned}
\operatorname{IV}(r-h) \cdot u^{1}+d^{2}+u^{3} & \equiv \mathrm{I} \cdot u_{\mathrm{R}}+d_{\mathrm{G}}+u_{\mathrm{B}}=\mathrm{II} \cdot d_{\mathrm{R}}+u_{\mathrm{G}}+u_{\mathrm{B}}=\mathrm{III} \cdot u_{\mathrm{R}}+u_{\mathrm{G}}+d_{\mathrm{B}}, \\
A & =\frac{1}{3}\left(u^{1}+d^{2}+u^{3}\right)=\frac{0}{3}=0 \\
& =\frac{+2}{3}+\frac{-13}{3}+\frac{+11}{3}=\frac{-16}{3}+\frac{+5}{3}+\frac{+11}{3}=\frac{+2}{3}+\frac{+5}{3}+\frac{-7}{3},
\end{aligned}
$$

example of $n^{0}$

$$
\begin{aligned}
\mathrm{IV}(r-h) \cdot u^{1}+d^{2}+d^{3} & \equiv \mathrm{I} \cdot u_{\mathrm{R}}+d_{\mathrm{G}}+d_{\mathrm{B}}=\text { II. } d_{\mathrm{R}}+d_{\mathrm{G}}+u_{\mathrm{B}}=\text { III. } d_{\mathrm{R}}+u_{\mathrm{G}}+d_{\mathrm{B}}, \\
A & =\frac{1}{3}\left(u^{1}+d^{2}+d^{3}\right)=\frac{-18}{3}=-6 \\
& =\frac{+2}{3}+\frac{-13}{3}+\frac{-7}{3}=\frac{-16}{3}+\frac{-13}{3}+\frac{+11}{3}=\frac{-16}{3}+\frac{+5}{3}+\frac{-7}{3},
\end{aligned}
$$

If array $q_{\mathrm{RGB}}$ is defined as a vector (26)

$$
q_{\mathrm{RGB}} \equiv\left(q_{\mathrm{R}}, q_{\mathrm{G}}, q_{\mathrm{B}}\right)=\vec{A}\left(q_{i}\right)
$$

Then the next two tables are constructed from CSDF, which may offer some heuristic search for classification of particle mass.

Table 6. Mass Values Comparison between Prediction and Experiment for proton and neutron [3].

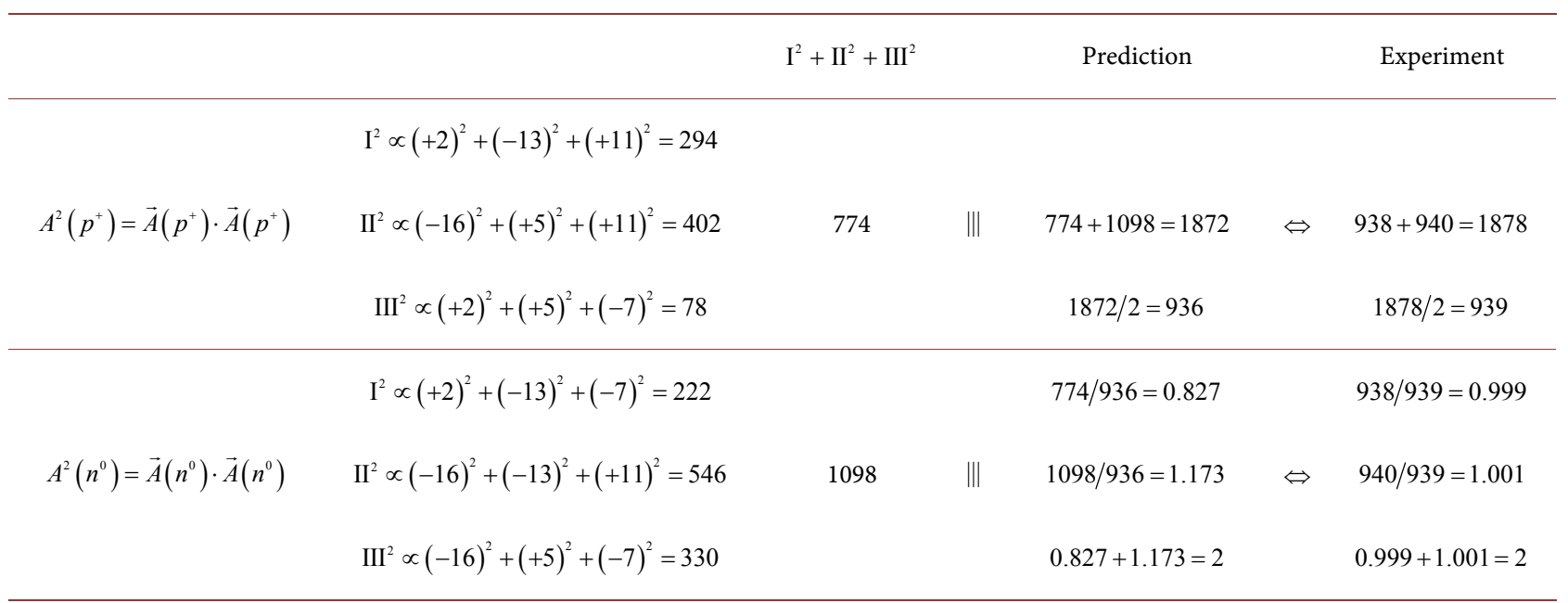


Table 7. Comparison between $A^{2}(q) / A^{2}(u)$ and $M(q) / M(u)$ of three generations of quarks (ref: diagram CSDF).

\begin{tabular}{|c|c|c|c|}
\hline$A^{2}(q)=q_{\mathrm{R}} q_{\mathrm{R}}+q_{\mathrm{G}} q_{\mathrm{G}}+q_{\mathrm{B}} q_{\mathrm{B}}$ & $A^{2}(q) / A^{2}(u)$ & $\mathrm{Mev} / \mathrm{c}^{2}(q) \Rightarrow M(q) / M(u)$ & $q$ \\
\hline$A^{2}(t) \propto(+38)^{2}+(+41)^{2}+(+47)^{2}=5334$ & $\Rightarrow 5334 / 150=35.56$ & $173 \times 10^{3} \Rightarrow 7521.7$ & $t$ \\
\hline$A^{2}(c) \propto(+20)^{2}+(+23)^{2}+(+29)^{2}=1770$ & $\Rightarrow 1770 / 150=11.8$ & $1.275 \times 10^{3} \Rightarrow 554.3$ & $c$ \\
\hline$A^{2}(u) \propto(+2)^{2}+(+5)^{2}+(+11)^{2}=150$ & $\Rightarrow 150 / 150=1$ & $2.3 \times 10^{0} \Rightarrow 1$ & $u$ \\
\hline$A^{2}(d) \propto(-16)^{2}+(-13)^{2}+(-7)^{2}=474$ & $\Rightarrow 474 / 150=3.16$ & $4.8 \times 10^{0} \Rightarrow 2.1$ & $d$ \\
\hline$A^{2}(s) \propto(-34)^{2}+(-31)^{2}+(-25)^{2}=2742$ & $\Rightarrow 2742 / 150=18.28$ & $95.0 \times 10^{\circ} \Rightarrow 41.3$ & $s$ \\
\hline$A^{2}(b) \propto(-52)^{2}+(-49)^{2}+(-43)^{2}=6954$ & $\Rightarrow 6954 / 150=46.36$ & $4.18 \times 10^{3} \Rightarrow 1817.4$ & $b$ \\
\hline
\end{tabular}

In Table 6 and Table 7, $A^{2}\left(p^{+}\right) A^{2}\left(n^{0}\right)$ and $A^{2}(q)$ are the scalar products of $\vec{A}\left(q_{i}\right)$ (26). The masses of particles (both proton, neutron and quarks) are supposed to be proportional to the scalar products from their corresponding CSDF.

\section{Conclusions}

In this paper we have pointed links between flavour quarks and colour quarks in math frame STS, Spin Topological Space: the flavour viewed as a number, named as flavour quantum number $A_{j, k}\left(q_{i}\right)$ and the colour viewed as an array, named as colour spectral line array $q_{\mathrm{RGB}}$ consist of three colour quantum numbers $q_{\mathrm{R}}, q_{\mathrm{G}}$ and $q_{\mathrm{B}}$ or $A\left(q_{\mathrm{R}}\right), A\left(q_{\mathrm{G}}\right)$ and $A\left(q_{\mathrm{B}}\right)$. The former is even number, the latter are third-fractions. When one thinks $I_{3}\left(q_{\mathrm{R}}\right), I_{3}\left(q_{\mathrm{G}}\right)$ and $I_{3}\left(q_{\mathrm{B}}\right)$ as three distinct angular momentums respectively, using momentum addtion of three-body, one can construct a variety of baryons.

In contrast to SM, mesons only are made of quark and antiquark, it becomes more complex, as now gluon joins into meson mechanism. In account of what happened in colour spectral line array $q_{i} \bar{q}_{\bar{j}} g_{k}$ when $i \neq j, k \neq 0$, many efforts are needed, after all, so much is not fully understood.

Perhaps CSDF, Colour Spectrum Diagram of Flavour is an essential conception for us to realize what flavour and colour of quarks are.

\section{Ackonwledgements}

The author thanks my intimate friend, Xin Mao, for valuable discussions and encouragements, and grateful to Jia Guan who helpfully supportes this paper Latex version.

\section{Conflicts of Interest}

The author declares no conflicts of interest regarding the publication of this paper. 


\section{References}

[1] Gell-Mann, M. (1964) Physics Letters, 8, 214-215. https://doi.org/10.1016/S0031-9163(64)92001-3

[2] Zweig, G. (1964) An SU(3) Model for Strong Interaction Symmetry and Its Breaking Version 2. CERN-TH-412. Version 1 Is CERN Preprint 8182/TH.401.

[3] Martin, B.R. and Shaw, G. (2017) Particle Physics. 4th Edition, John Wiley \& Sons, Ltd., The Atrium, Southern Gate, Chichester, West Sussex, PO19 8SQ, Kingdom.

[4] Ren, S.X. (2011) The Third Kind of Particle. Shanghai Electronic Publishing Limited, Shanghai.

[5] Ren, S.X. (2012) The Third Kind of Particle. Modern Culture Publisging House, Hong Kong.

[6] Ren, S.X. (2015) Interaction of the Origins of Spin Angular Momentum. China Science Literature Publisging House, Hong Kong.

[7] Ren, S.X. (2017) 642 WE-Heraeus-Seminar, Non-Hermitian Hamiltonians in Physics: Theory and Experiment. Physikzentrum Bad Honnef Germany. 